\title{
IMPLANTAÇÃO DO BUSINESS INTELLIGENCE PARA GESTÃO dA INFORMAÇÃO EM UNIDADES HOSPITALARES
}

\author{
Implementation of business intelligence in the \\ information management of hospital units
}

\author{
Annanda Letícia Unicki Ribeiro', Vivian Patrícia Raksa ${ }^{2}$
}

1 Administradora na Superintendência de Unidades Hospitalares Próprias da Secretaria de Estado da Saúde do Paraná. ORCID: https://orcid.org/0000-0001-7485-9289.

2 Mestre em Planejamento e Governança Pública pela Universidade Tecnológica Federal do Paraná. Enfermeira na Superintendência de Unidades Hospitalares Próprias da Secretaria de Estado da Saúde do Paraná. ORCID: http://orcid.org/0000-0001-7112-9934.

CONTATO: Annanda Letícia Unicki Ribeiro | E-mail: annandaleticia@hotmail.com

COMO CITAR Ribeiro ALU, Raksa VP. Implantação do business intelligence para gestão da informação em unidades hospitalares. R. Saúde Públ. 2018 Dez;1(2):152-160

\begin{tabular}{lll}
\hline Ca) CoPYRIGHT Esta obra é disponibilizada nos termos da Licença Creative Commons - 4. 0 \\
Internacional. É permitida a reprodução parcial ou total desta obra, desde que citada a fonte.
\end{tabular}

Relato de Experiência vencedor do $3^{\circ}$ Prêmio Inova Saúde Paraná, realizado durante o $4^{\circ}$ Congresso Paranaense de Saúde Pública/Coletiva (2018), no eixo temático "Ciência, Tecnologia e Inovação em Saúde".

RESUMO Este relato de experiência tem como objetivo apresentar a implantação da ferramenta Business Intelligence (B.I) para a gestão da informação em hospitais próprios do estado do Paraná. Os resultados da produtividade hospitalar são repassados por meio de uma planilha de controle chamada de sistema de informação gerencial (SIG), sendo ela composta por quatro modalidades. Cada hospital gera uma planilha com um alto volume de dados, fato que dificulta a comparação dos resultados entre os hospitais. Diante desta barreira, desenvolveuse o B.I o qual possibilita analisar dados de diferentes unidades e períodos. Conclui-se que o desenvolvimento desta ferramenta, permite o acesso sistematizado aos dados, padronização das planilhas, celeridade na tomada de decisão, acompanhamento e planejamento dos resultados.

PALAVRAS-CHAVE: Sistemas de Informação. Base de Dados. Rede de Comunicação. Processamento Automatizado de Dados. 
ABSTRACT This experience report aims to present the implementation of the Business Intelligence tool (B.I) for information management in hospitals of the state of Paraná. The results of hospital productivity are passed through a control spreadsheet called management information system (SIG), which consists of four modalities. Each hospital generates a spreadsheet with a high volume of data, which makes it difficult to compare the results among hospitals. Thus, B.I was developed, allowing data analysis from different units and periods. It is concluded that the development of this tool allows systemic access to data, standardization of spreadsheets, speed in decision making, monitoring and planning of results.

KEYWORDS: Information Systems. Data Base. Communication Network. Automated Data Processing.

\section{INTRODUÇÃO}

$\mathbf{0}$

Estado do Paraná possui 15 unidades hospitalares próprias e 4 hospitais universitários estaduais, sendo que a Superintendência de Unidades Próprias (SUP), localizada na Secretaria de Saúde do Estado do Paraná (SESA), é responsável pela gestão, acompanhamento e avaliação dos hospitais próprios e a SESA juntamente com a Secretaria da Ciência, Tecnologia e Ensino Superior (SETI) realizam a gestão compartilhada dos hospitais universitários. Desta forma, a rede própria é composta por 19 hospitais que estão localizados em território paranaense conforme apresentado na Figura 1.

Figura 1 Mapa da Unidades Hospitalares Próprias do Estado do Paraná.

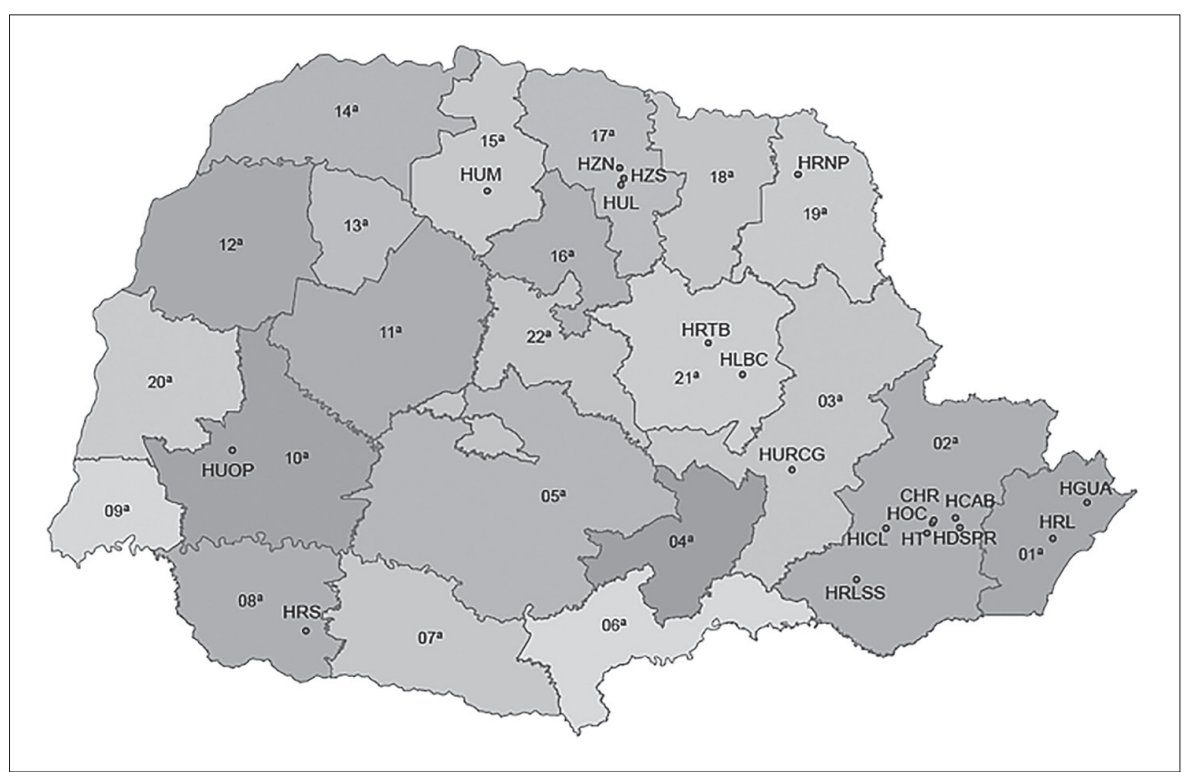

Fonte: Arcgis SESA 
Os 19 hospitais possuem perfil assistencial distinto, o que dificulta a coleta e análise das informações de produtividade, visto que os dados publicizados no Tabwin / DataSUS são referentes ao que é faturado nas instituições hospitalares e podem não refletir a realidade. Com o objetivo de superar esta limitação, em 2011 a SUP, desenvolveu e implantou o sistema de informações gerenciais (SIG), que visa coletar os dados da produtividade hospitalar, a fim de acompanhar e avaliar os resultados dos hospitais que compõem a rede própria.

O SIG permite a coleta, armazenamento, processamento de dados e disponibiliza informações para todos os níveis organizacionais. Desta forma, deve prover informações de cunho estratégico e operacional'.

O SIG utilizado pela SUP/SESA é estruturado no Excel. A primeira versão em 2011 foi composta por 94 indicadores, sendo revisada anualmente, o que culminou em uma versão em 2018 com 125 indicadores. O SIG 2018 está estruturado em quatro modalidades: produtividade hospitalar, informações financeiras, gestão de pessoas e o programa hospitais do Paraná (que se refere a dados sobre a qualidade e segurança do paciente). Mensalmente cada hospital envia a planilha com o resultado dos seus indicadores, o que permite a composição do banco de dados.

Com a utilização da ferramenta ao longo dos anos foi possível o estabelecimento de um banco de dados consistentes sobre a produtividade hospitalar, no entanto observou-se uma dificuldade na compilação dos resultados desses 19 hospitais, assim como na análise comparativa em um período de tempo ou entre instituições.

No final de 2017 o banco de dados contava com mais de 10.000 elementos, o que gerava dificuldades na unificação da informação, assim como eram evidenciadas planilhas sem padronização devido à movimentação dos dados pelos usuários, agravados pela ausência de pontualidade na entrega das planilhas. Fatos estes, que não agregavam no processo para uma rápida tomada de decisão.
Com o objetivo de otimizar este processo de trabalho, a SUP firmou parceria com a Companhia de Tecnologia da Informação e Comunicação do Paraná (Celepar), para desenvolvimento de um programa que permitisse a análise comparativa e gráfica dos indicadores apresentados pelos hospitais da rede própria do Estado. Para tal, foi utilizado o conceito de Business Intelligence (B.I), que "é um processo que envolve métodos, técnicas, tecnologias, pessoas, informações, fontes de informações, métricas, ferramentas, etc" 2.

O B.I baseia-se em agrupar informações de diversas fontes e apresentá-las de forma unificada e sob uma métrica comum, gerando um conjunto de conceitos e métodos para melhorar o processo de tomada de decisão das empresas, utilizando-se de sistemas fundamentos em dimensões e fatos ${ }^{3}$.

Desta forma, propõe-se relatar a experiência na utilização da ferramenta B.I para compilar e analisar os dados de produtividade dos hospitais próprios do Estado do Paraná, transformando dados em informações imprescindíveis à tomada de decisão e gestão hospitalar. O B.I precisa disponibilizar a informação correta, para os envolvidos no tempo necessário, contribuindo para decisões assertivas para visar a relação custo-benefício ${ }^{4}$.

\section{METODOLOGIA}

Trata-se de um relato de experiência que "deve ser redigido de maneira que possibilite a comprovação dos procedimentos, das técnicas e dos resultados obtidos"5. Desta forma, este relato tem como objetivo apresentar o processo de implantação da ferramenta B.l, como solução na integração dos dados de diversas unidades hospitalares. O mesmo ainda expõe as etapas realizadas pela SUP para adequação do sistema e as melhorias proporcionadas por ele. 


\section{RELATO DA}

\section{EXPERIÊNCIA E DISCUSSÃO}

O controle das informações por meio do SIG é dividido em quatro modalidades, sendo elas: produção hospitalar, gestão de pessoas, financeiro e o programa da qualidade e segurança do paciente. Os dados são atualizados mensalmente e geram automaticamente os indicadores. Possui campo para justificativas quanto a atrasos, variações ou qualquer outro fator relevante. Desta forma, é possivel manter um histórico relativo à evolução de cada indicador e ainda consultar a informação do exercício já encerrado. Outra facilidade ao usuário refere-se ao comentário disponível em cada dado / indicador, onde é possível consultar o conceito do item a ser informado, assim como deve ser preenchido, ou seja, as regras a serem seguidas.

A modalidade produção hospitalar é composta pela quantidade de leitos ativos, número de internações, média de permanência, taxa de ocupação, número de cirurgias, número de consultas em pronto-socorro, pronto-atendimento ou ambulatório, quantidade de terapias, número de refeições servidas, número de roupas lavadas, entre outros indicadores de produtividade.

Na modalidade Gestão de Pessoas, são controlados o número de servidores e profissionais terceiros lotados na unidade, o total de horasextras, horas trabalhadas, especialidades médicas, afastamentos, quantidade de plantonistas, e as movimentações entre entradas e saídas de servidores.

$\mathrm{Na}$ perspectiva financeira, os indicadores dispostos no SIG se referem ao total do faturamento ambulatorial, de procedimentos de alto custo e internações. Além de dados como receitas e despesas do Sistema Único de Saúde (SUS), receitas e despesas dos convênios e os custos hospitalares.

A quarta divisão diz respeito ao Programa Hospitais do Paraná, que visa à qualidade e segurança do paciente em ambientes hospitalares.
Nesta, é possível analisar indicadores de incidentes e eventos adversos e protocolos de identificação do paciente, higienização das mãos, cirurgia segura, protocolos de queda, lesão por pressão, segurança da prescrição, uso e administração de medicamentos, além de dados sobre a satisfação dos pacientes internados.

Com a utilização da ferramenta ao longo dos anos foi possível o estabelecimento de um banco de dados consistentes sobre a produtividade hospitalar, contudo dificuldades eram evidenciadas na compilação dos resultados, devido aos diferentes sistemas operacionais, assim como na análise comparativa em um período de tempo ou entre instituições.

No gráfico 1 é possível verificar a evolução do quantitativo de indicadores analisados ano a ano.

Em 2011 foram compilados 1.128 dados englobando todos os hospitais da rede própria. Ao final de 2017, evidencia-se um crescimento de $37,24 \%$, passando a 1.548 dados coletados no ano. Desta forma, o volume de informações a serem compiladas exigia um sistema que comportasse tal quantidade de dados.

Após o estabelecimento da parceria entre a SUP e a Celepar, a qual detém a expertise no B.I, foram alinhados os fatores críticos de sucesso para o desenvolvimento desta ferramenta.

Para atender ao objetivo de desenvolver um instrumento com informações rápidas, foram realizados ajustes como a padronização das planilhas e dos indicadores, uma vez que o B.l realiza a leitura sistêmica dos dados.

A padronização dos indicadores foi outro ajuste realizado, pois entre os hospitais existiam diferenciações entre os dados solicitados. 0 que era necessário para uma unidade hospitalar não necessariamente seria para outra. Desta forma, para solucionar o problema foi definido que todos os dados comporiam a planilha SIG, no entanto, o preenchimento aconteceria apenas a quem de fato o aplicasse na instituição. Além das padronizações no formato das planilhas e dos indicadores, foram 
Gráfico 1 Total de Indicadores por ano

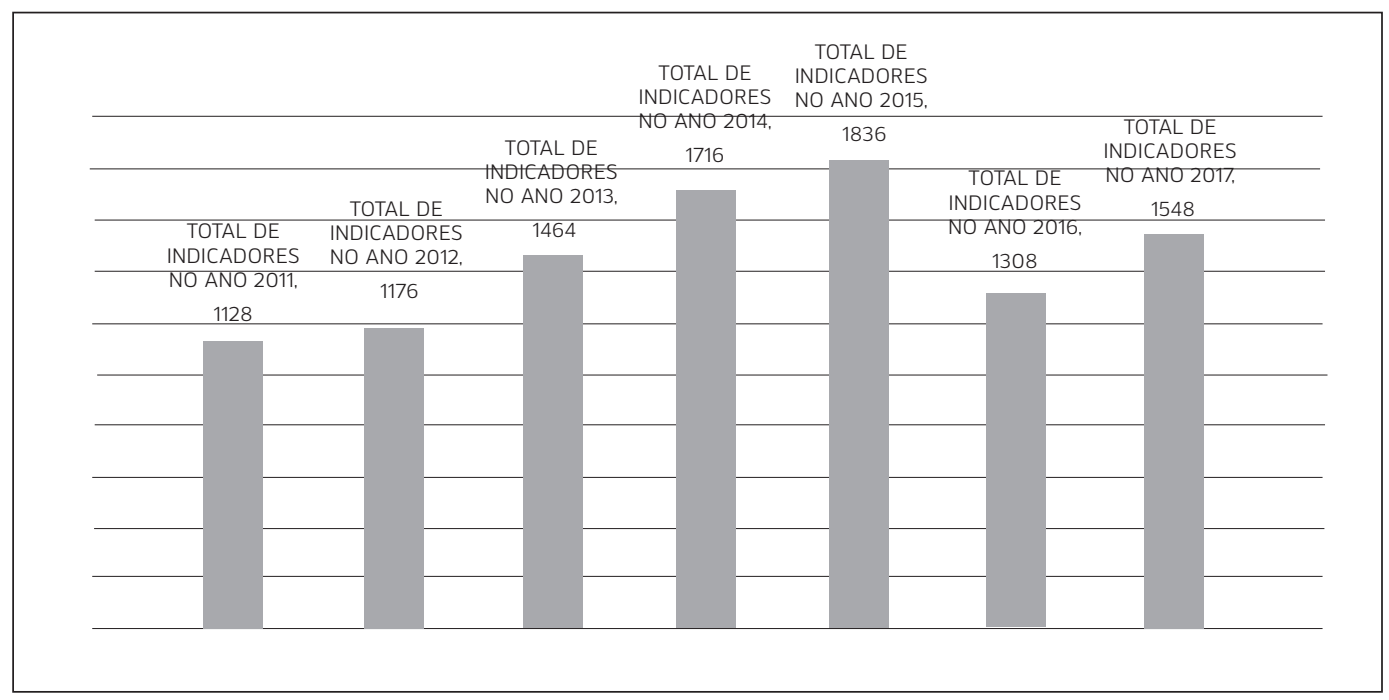

Fonte: Elaborado pelos autores (2018)

realizados treinamentos entre os usuários para que estes compreendessem a importância do preenchimento do dado e seus reflexos.

O prazo de entrega das planilhas foi outro fator apresentado aos usuários. A exigência de pontualidade na entrega dos dados também foi uma mudança aplicada no processo de desenvolvimento da ferramenta B.I do SIG para que ao analisar os dados, o usuário pudesse comparar os resultados entre diferentes unidades hospitalares. Conforme Meireles': "Um Sistema de Informações Gerenciais é abrangente e produz todas as informações necessárias para todos os níveis dentro da organização. Para ser uma ferramenta útil, a informação deve ser completa, precisa e apropriada para a tarefa e a pessoa destinada, e deve ser entregue com pontualidade. A informação fornecida deve se equiparar à necessária para a tomada de decisão".

Caso a planilha do SIG não fosse entregue, não seria possível a análise dos resultados. Assim sendo, criou-se um fluxo onde cada unidade hospitalar indicou um usuário chamado de "pessoachave" que é o responsável pelo cumprimento dos prazos e a entrega dos resultados. Além disso, este grupo de "pessoas-chave" passou a receber as orientações na medida em que divergências eram identificadas no preenchimento das planilhas.

O ganho de tempo e a fidedignidade da informação são melhorias no processo, dado que o B.I apresenta as variações dos resultados o que permite ao usuário da ferramenta solicitar justificativas alusivas a estas diferenças. Na hipótese de identificação de um erro de lançamento, ele é corrigido e o sistema passa a apresentar as informações em conformidade com a realidade do hospital. Se a distorção do resultado for mantida, a planilha dispõe de campos para justificativas, o que proporciona a rastreabilidade das mudanças ao longo dos períodos.

Com essas mudanças foi possível melhorar o planejamento, controle, monitoramento e avaliação dos resultados apresentados pelas Unidades Hospitalares Próprias do Estado do Paraná, o que está alinhado com os conceitos de SIG trazidos por Rodrigues e Gedres6: "O sistema de informação gerencial dá suporte às funções de planejamento, controle e organização de uma 
empresa, fornecendo informações seguras e em tempo hábil para tomada de decisão. 0 sistema de informação gerencial é representado pelo conjunto de subsistemas, visualizados de forma integrada e capaz de gerar informações necessárias ao processo decisório".

Aronson, King e Turban afirmam que:

"Os principais objetivos do BI são permitir o acesso interativo aos dados (às vezes, em tempo real), proporcionar a manipulação desses dados e fornecer aos gerentes e analistas de negócios a capacidade de realizar a análise adequada. Ao analisarem dados, situações e desempenhos históricos e atuais, os tomadores de decisão conseguem valiosos insights que podem servir como base para decisões melhores e mais informadas. O processo do $\mathrm{Bl}$ baseia-se na transformação de dados em informações, depois em decisões e finalmente em ações. O B.I contribuiu para a tomada de decisão rápida, identificação de variações nos dados informados, facilidade na visualização do dado, comparação entre diversos hospitais e diferentes períodos, além de proporcionar agilidade na consulta aos indicadores."7

Nesse sentido, a ferramenta implementada neste relato contribui no ganho de tempo, uma vez que todos os dados são compilados sendo necessário realizar os filtros dos indicadores a serem analisados. O usuário destina o tempo nos resultados relevantes o que permite uma otimização no processo de análise e, caso necessário, intervenções voltadas às divergências identificadas. .

Na Figura 2, descrevemos as etapas realizadas de cada um dos envolvidos no processo, desde a extração dos dados até o processamento do B.l:

Na Figura 3, apresentamos os resultados gráficos que a ferramenta permite. Na coluna à esquerda o usuário aplica os filtros e o sistema apresenta os dados de forma gráfica.

No B.I do SIG é possível comparar os resultados entre diferentes hospitais e exportá-los a fim de elaborar relatórios analíticos. A ferramenta permite analisar evoluções no que tange a produtividade hospitalar, assim como os dados financeiros, da qualidade e segurança do paciente e na gestão de pessoas ao longo do tempo. Além disso, os resultados são visualizados em seis diferentes formas gráficas como barras, linhas, grades, radar e funil. Estas propiciam decisões rápidas em decorrência da facilidade na compressão do resultado apresentado.

$\mathrm{Na}$ Figura 4, ampliamos a imagem para demonstrar exemplos de filtros que podem ser realizados na consulta do B.I do SIG.

Figura 2. Etapas do B.I.

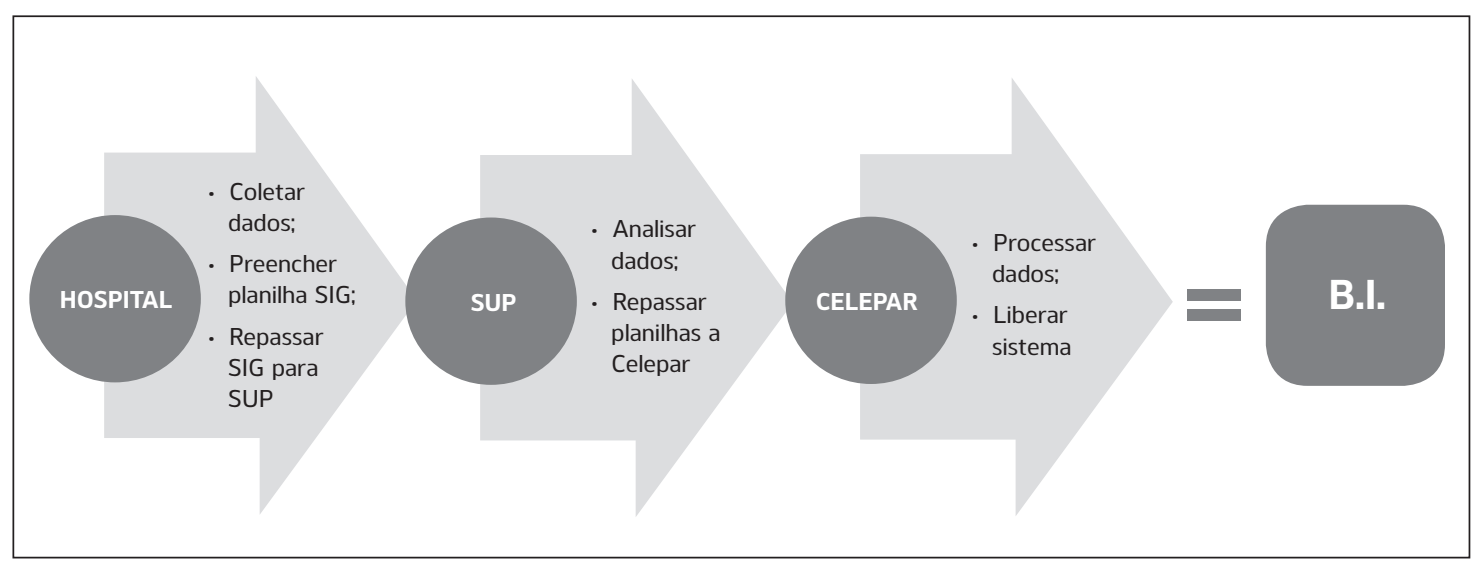

Fonte: Elaborado pelos autores (2018). 
Figura 3. Imagem da ferramenta B.I do SIG

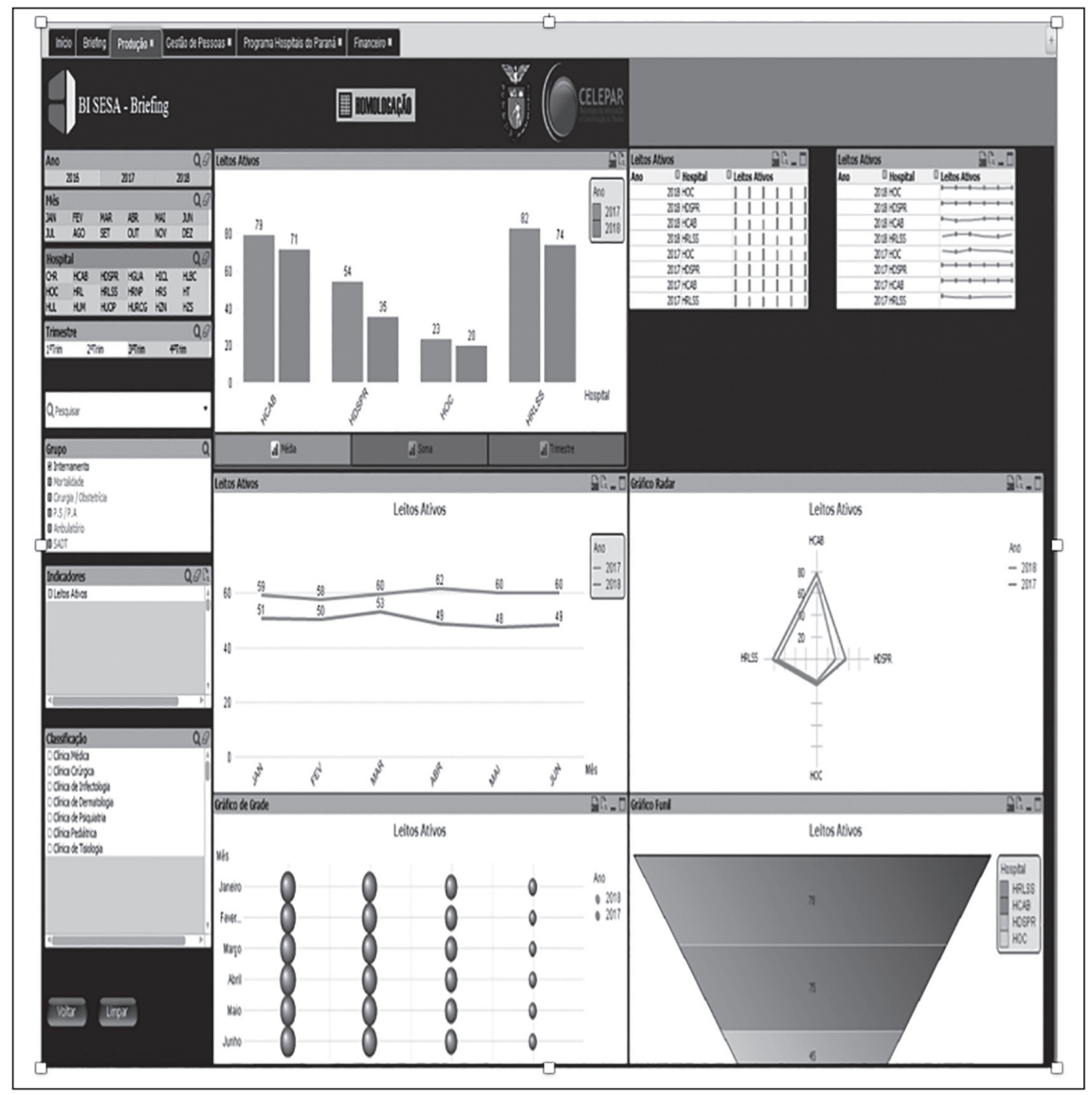

Fonte: Elaborado pelos autores e a Celepar (2018)

Os filtros disponíveis para seleção se dividem em grupos com intuito de facilitar a organização dos indicadores. Nestes, o usuário pode optar pelo período que deseja visualizar no gráfico, ano, mês ou trimestre, além de indicar o grupo ao qual o indicador pertence. Após deverá escolher qual indicador tem preferência e então o resultado gráfico será apresentado na tela do sistema. Além disso, o B.I do SIG permite um detalhamento do resultado, ou seja, é possível selecionar o indicador por tipo de clínica, faixa etária ou até mesmo por tipo de exame realizado. Desta forma, o dado pode ser apresentado de forma sistêmica ou direcionada para atender a necessidade do usuário.

\section{CONCLUSÃO}

As informações da produtividade hospitalar são disponibilizadas pelo Ministério da Saúde, no entanto, estas não refletem a realidade dos hospitais próprios do Estado do Paraná, o que gerou a necessidade desenvolvimento de uma ferramenta própria para coleta e análise de dados, sendo instituído o SIG. Ao longo da implantação, este demonstrou eficiência na coleta de dados, mas não permitiu a análise conjunta e em tempo oportuno dos resultados de todas as unidades hospitalares que compõem a rede própria.

Desta forma, desenvolveu-se o B.I, utilizando 
Figura 4. Imagem de alguns dos filtros que podem ser selecionados no B.I do SIG.

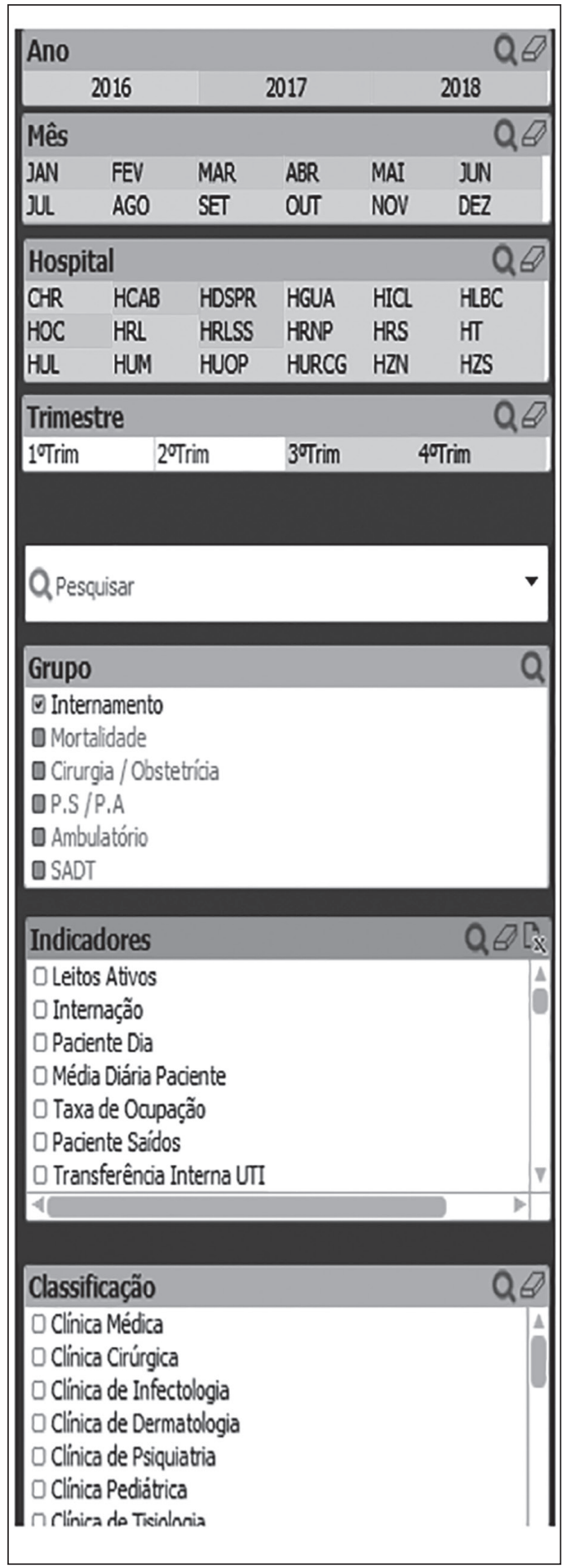

Fonte: Elaborado pelos autores e a Celepar (2018) o banco de dados do SIG, o que permite uma análise estruturada das informações, além de permitir a visualização gráfica da produtividade hospitalar, que otimiza o acesso à informação e a tomada rápida de decisão. Ademais, permite o acompanhamento dos resultados e das variações dos indicadores preenchidos pelos hospitais da rede própria do Paraná. A implantação desta ferramenta permite a transformação eficiente de dados brutos em informações estratégicas para a gestão hospitalar.

Além da agilidade na tomada de decisões, há também melhoria no processo de planejamento de metas, acompanhamento e avaliação das instituições. O controle torna-se efetivo visto que as divergências são identificadas na medida em que há atualizações de dados. Os hospitais são monitorados mensalmente e avaliados conforme os resultados alcançados.

Em função dos fatos mencionados, obtevese um ganho em relação ao tempo de análise dos dados uma vez que o usuário do B.I do SIG pode destinar as avaliações aos itens de maior relevância e programar apreciações a itens de menor impacto conforme a necessidade de cada hospital.

Por fim, percebe-se que a ferramenta contribui para a gestão hospitalar, pois reflete de forma rápida e eficaz os resultados realizados em cada unidade da rede própria.

\section{REFERÊNCIAS}

1. Meireles M. Sistemas de informação: quesitos de excelência dos sistemas de informações operativos e estratégicos. São Paulo: Arte \& Ciência; 2004

2. Loh S. BI na era do big data para cientistas de dados: indo além de cubos e dashboards na busca pelos porquês, explicações e padrões. Porto Alegre: Stanley Loh; 2014. 252 p.

3. Braghittoni R. Business Intelligence: implementar do jeito certo e a custo zero. [local desconhecido]: Editora Caso do Código; 2017. $249 \mathrm{p}$.

4. Sezões C., Oliveira J., Baptista M. Business Intelligence. Porto: Sociedade Portuguesa de Inovação; 2006. 
5. Freitas EC, Prodanov CC. Metodologia do trabalho científico: métodos e técnicas da pesquisa e do trabalho acadêmico. 2. ed. Rio Grande do Sul: Feevale; 2013. 276 p.

6. Rodrigues LS, Gedres CAP. Tópicos emergentes do sistema de informação gerencial [Internet]. [local desconhecido]:Administradores-O Portal da Administração; 2008. [citado 2018 Ago 28]. Disponível em: http://www.administradores. com.br/artigos/tecnologia/sistema-de-informacaogerencial/23741.

7. Aronson JE, King D. Sharda R, Turban E. Business intelligence: um enfoque gerencial para a inteligência do negócio. São Paulo: Bookmanm; 2009. 254 p. 